\title{
Follicular mucinosis: an important differential diagnosis of leprosy in an endemic area*
}

\author{
Danielle Cristine Westphal ${ }^{1}$ \\ Petra Pereira de Souza ${ }^{1}$ \\ Antônio Pedro Mendes Schettini ${ }^{1}$
}

\author{
Silmara Navarro Pennini ${ }^{1}$ \\ Gustavo Ávila Maquiné ${ }^{2}$ \\ Mônica Santos ${ }^{1,3}$
}

DOI: http:/ /dx.doi.org/10.1590/abd1806-4841.20153450

\begin{abstract}
Primary follicular mucinosis is a rare dermatosis characterized by the accumulation of mucin in the follicular epithelium and sebaceous glands. Clinically, it is characterized by the presence of papules or wellcircumscribed and infiltrated plaques. In this paper, we report the case of a female patient, seven years old, evolving for three months with an asymptomatic, erythematous and infiltrated plaque located in the chin region. The research of thermal, pain and tactile sensitivity was inconclusive. Histological findings confirmed the diagnosis of follicular mucinosis. There was regression of the lesion with the use of medium potency topical corticosteroids for 20 days. The pathogenesis of follicular mucinosis remains unknown, being in some cases associated with lymphoproliferative disorders. In endemic areas of leprosy, isolated and infiltrated follicular mucinosis lesions should be further differentiated from leprosy.
\end{abstract}

Keywords: Diagnosis, differential; Leprosy, tuberculoid; Mucinosis, follicular

\section{INTRODUCTION}

Follicular mucinosis (FM) is a rare dermatosis whose etiology is not fully understood, characterized by the accumulation of mucin in the follicular epithelium and sebaceous glands. It was described for the first time by Pinkus et al., in 1957, who named it mucinous alopecia. ${ }^{1}$ Since this alopecia is not seen in all cases, being best observed in areas with terminal hairs, Jablonska et al. proposed in 1959 to name it follicular mucinosis, the designation more commonly adopted nowadays. ${ }^{2}$ It is clinically characterized by the presence of well-delimited, erythematous or brownish-erythematous, infiltrated papules or plaques. Follicular keratosis or areas of alopecia may also be observed. Other less common presentations have been already described, with acneiform, eczematous, cystic or nodular lesions. ${ }^{3}$ FM may also present itself as an exclu- sive form or in association with diverse benign or malignant conditions, such as hematological neoplasms, infections and autoimmune diseases. ${ }^{4}$ In this work, we report a case of a child with an infiltrated erythematous plaque in the chin region, whose clinical presentation constitutes an important differential diagnosis of childhood tuberculoid leprosy, mainly in endemic areas of leprosy.

\section{CASE REPORT}

Female patient, seven years old, evolving for three months with asymptomatic cutaneous lesion located in the chin region. At the dermatological examination, an erythematous-brownish plaque, ovalshaped, well-circumscribed and infiltrated, measuring approximately $2.5 \mathrm{~cm}$ in its largest diameter was

Received on 14.02.2014

Approved by the Advisory Board and accepted for publication on 16.03.2014

Work performed at Fundação Alfredo da Matta (Fuam) - Manaus (AM), Brazil.

Financial Support: None.

Conflict of Interest: None.

Fundação Alfredo da Matta (Fuam) - Manaus (AM), Brazil

Secretaria Municipal de Sáude de Manaus - Manaus (AM), Brazil.

Universidade do Estado do Amazonas (UEA) - Manaus (AM), Brazil.

C2015 by Anais Brasileiros de Dermatologia 
noticed in the chin region (Figure 1). The evaluation of thermal, pain and tactile sensitivity was inconclusive. In light of the clinical picture, hypotheses of childhood tuberculoid leprosy and follicular mucinosis were raised. Bacilloscopy was negative. At the histological examination, reticular degeneration in the infundibulum of hair follicles was observed, forming large clear spaces, with lymphohistiocytic infiltrate around vessels and appendages and discrete collagen fibrosis. Alcian blue staining confirmed accumulation of mucin in hair follicles. Atypical lymphocytes or epidermotropism were not observed (Figure 2). Patient was treated with medium-potency topical corticoid during 20 days, evolving with complete remission of lesion (Figure 3). She remains under outpatient clinic follow-up, twelve months after the initial treatment, with no relapses.

\section{DISCUSSION}

Follicular mucinosis is a disorder histopathologically defined by the accumulation of mucin in hair follicles and sebaceous glands, which undergo epithelial reticular degeneration. It is believed that follicular keratinocytes are responsible for mucin production, as a response to the stimulus of cytokines released by perifollicular $\mathrm{T}$ lymphocytes. Its pathogenesis, however, remains unknown, even though the role of circulating immune complexes and cell immunity have already been considered. ${ }^{5}$ Classically, three forms are described: primary of short evolution; primary of pro-

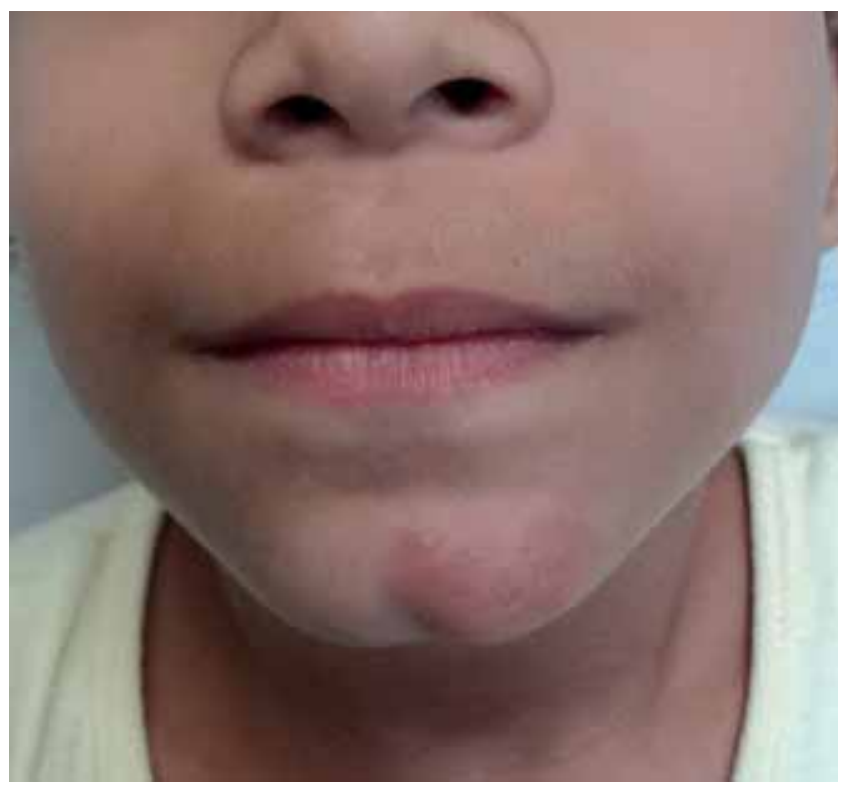

FIGURE 1: Erythematous-brownish oval-shaped plaque, well-circumscribed and infiltrated, measuring approximately $2.5 \mathrm{~cm}$ at its largest diameter, located in the chin region
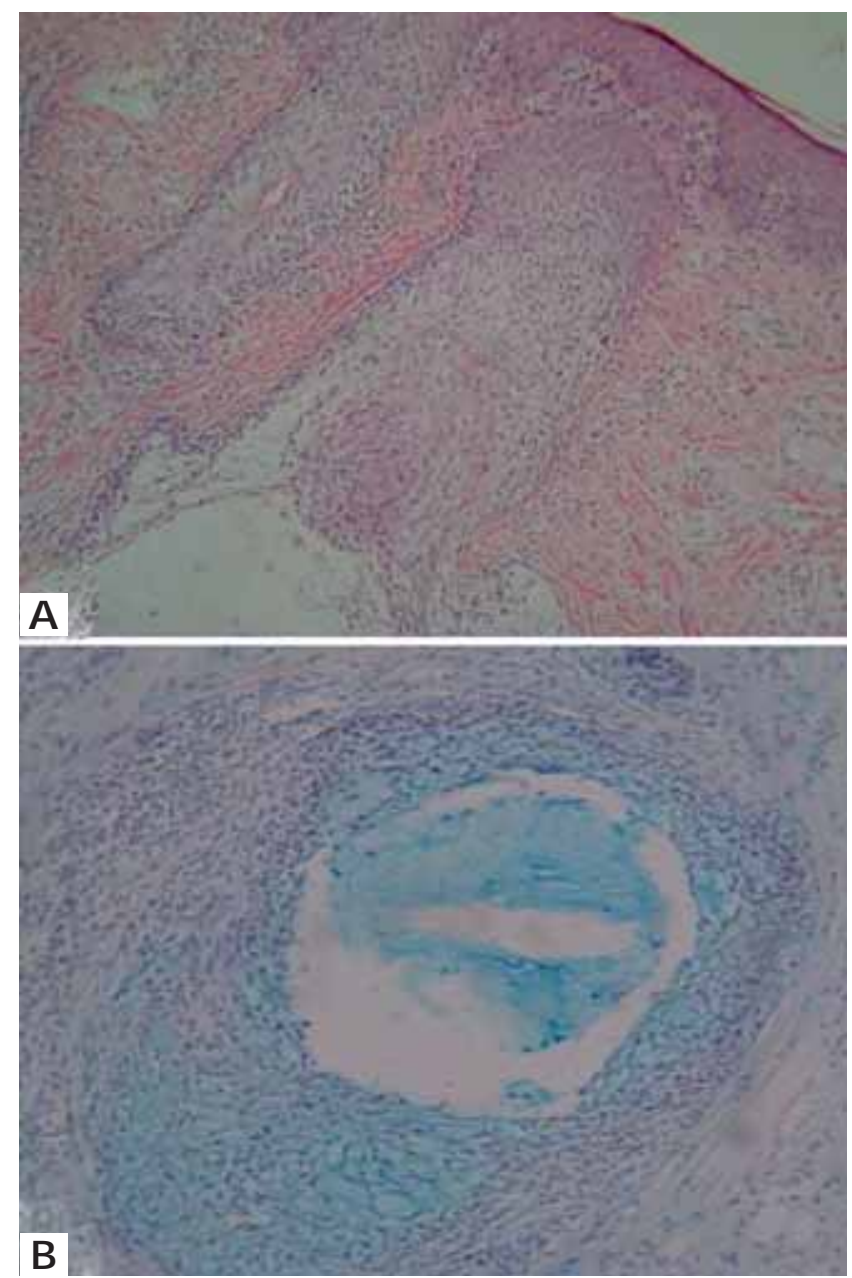

Figure 2: In hematoxilin-eosin staining, reticular degeneration in the infundibulum of hair follicles was observed, forming large clear spaces, with lymphohistiocytic infiltrate around vessels and appendages and discrete collagen fibrosis

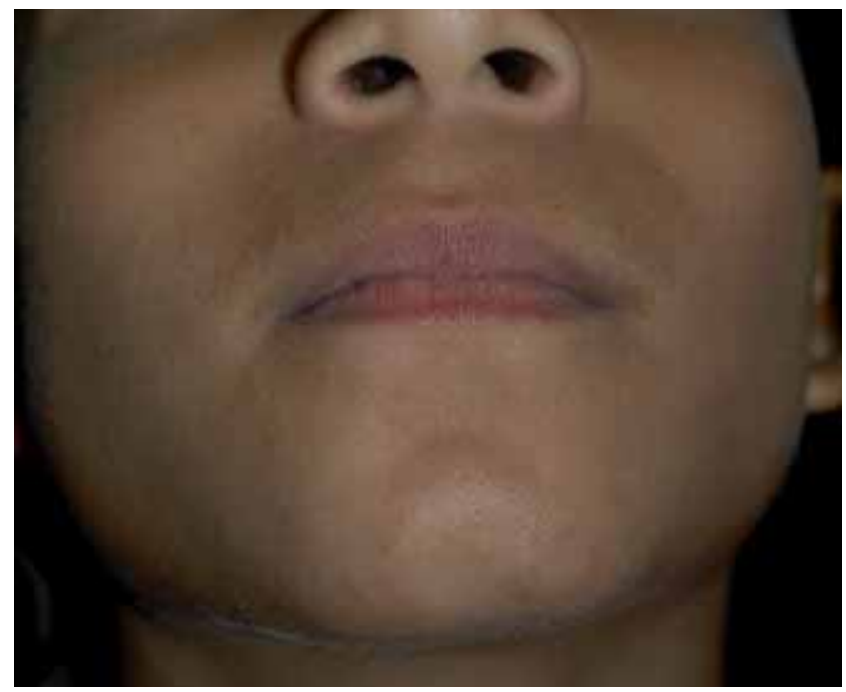

FIGURE 3: Complete remission of lesion after treatment with topical corticoid 
longed course; and secondary, associated with diverse benign or malignant conditions. Primary follicular mucinosis of short evolution affects children and young adults, presenting few lesions, limited to the head and neck regions, which may remit spontaneously with no relapses, similarly to the case presented. Primary FM of prolonged course occurs in older patients, manifesting itself with multiple lesions of chronic course, prone to relapses. In both cases there is usually no association with systemic diseases, although some of the latest studies have demonstrated the presence of histological characteristics of mycosis fungoides in patients with primary FM. ${ }^{6}$ Secondary mucinosis usually appears between the fourth and seventh decades of life, with multiple infiltrated plaques, which are commonly associated with systemic diseases, particularly lymphoproliferative malignancies. ${ }^{7}$ The creation of a new clinical subtype named reactive follicular mucinosis was recently proposed, where incidental histopathological findings may be observed in some dermatoses, with mucin deposits, such as hypertrophic lichen planus, striated lichen and melanocytic nevus. ${ }^{8}$ In the case presented, the importance of differential diagnosis between primary follicular mucinosis and Hansen's disease is emphasized, mainly in cases with presence of infiltrated plaque in patients who are from endemic Hansen's disease areas. Studies published in the literature show that, in endemic areas, the differential diagnosis between Hansen's disease and follicular mucinosis is not always easy and must be considered. In the case presented, the histological examination was essential for the diagnosis, since the inflammatory infiltrate composed of macrophages, perifollicular and perivascular lymphocytes, with no atypias or epidermotropism, rejected the diagnosis of childhood tuberculoid leprosy, confirming the diagnosis of FM. Besides Hansen's disease, primary FM must be differentiated from persistent reaction to insect bite, lymphocytoma and lymphocytic infiltrate, among others. ${ }^{9}$

Several therapeutic modalities have been described for primary FM, with the use of topical or intralesional corticosteroids, dapsone, antimalarial agents, oral retinoids, interferon alpha, minocycline and phototherapy. ${ }^{10}$ In the case presented, there was total remission of lesion with the use of medium potency topical corticoid for 20 days.]

\section{REFERENCES}

1. Pinkus H. Alopecia mucinosa: inflammatory plaques with alopecia characterized by root-sheath mucinosis. AMA Arch Derm. 1957;76:419-24.

2. Jablonska S, Chorzelski T, Lancucki J. Mucinosis follicularis. Hautarzt. 1959:10:27-33.

3. Bella-Navarro R, Martí-Fajardo N, Martín-Hernández JM, Jordá-Cuevas E. Mucinosis folicular en la infancia: aportación de un caso y revisión de la literatura. Actas Dermosifiliogr. 2012;103:335-6.

4. Alikhan A, Griffin J, Nguyen N, Davis DM, Gibson LE. Pediatric Follicular Mucinosis: Presentation, Histopathology, Molecular Genetics, Treatment, and Outcomes over an 11-Year Period at the Mayo Clinic. Pediatr Dermatol. 2013;30:192-8.

5. Fonseca APM, Bona SH, Fonseca WSM, Campelo FS, Rego PMM. Follicular mucinosis: literature review and case report. An Bras Dermatol. 2002;77:701-6.

6. Rongioletti F, De Lucchi S, Meyes D, Mora M, Rebora A, Zupo S, et al. Follicular mucinosis: a clinicopathologic, histochemical, immunohistochemical and molecular study comparing the primary benign form and the mycosis fungoidesassociated follicular mucinosis. J Cutan Pathol. 2010;37:15-9.

7. Zvulunov A, Shkalim V, Ben-Amitai D, Feinmesser M. Clinical and histopathologic spectrum of alopecia mucinosa/follicular mucinosis and its natural history in children. J Am Acad Dermatol. 2012;67:1174-81.

8. Blakey BL, Gratrix ML. Reactive Benign Follicular Mucinosis: a report of two cases. Cutis. 2012; 89:266-268.

9. Joshi R, Gopalani V. Alopecia mucinosa responding to antileprosy treatment: are we missing something? Indian J Dermatol. 2013;58:227-31.

10. Kim KR, Lee JY, Kim MK, Yoon TY. Successful Treatment of Recalcitrant Primary Follicular Mucinosis with Indomethacin and Low-dose Intralesional Interferon Alpha. Ann Dermatol. 2009;21:285-7.

\author{
M AILING ADDRESS: \\ M ônica Santos \\ A venida Codajas, 25 \\ Cachoeirinha \\ 69065-130 - M anaus - A M \\ Brazil \\ E-mail:m.n.souza.santos@gmail.com
}

H ow to cite this article: Westphal DC, Pennini SN, Souza PP, Maquiné GA, Schettini APM, Santos M . Follicular mucinosis: an important differential diagnosis of leprosy in an endemic area . An Bras Dermatol. 2015;90 (3 Suppl 1):S147-9. 